\title{
Arithmetical aspects of certain functional equations
}

by

\author{
Lutz G. Lucht (Clausthal)
}

The classical system of functional equations

$$
\frac{1}{n} \sum_{\nu=0}^{n-1} F\left(\frac{x+\nu}{n}\right)=n^{-s} F(x) \quad(n \in \mathbb{N})
$$

with $s \in \mathbb{C}$, investigated for instance by Artin (1931), Yoder (1975), Kubert (1979), and Milnor (1983), is extended to

$$
\frac{1}{n} \sum_{\nu=0}^{n-1} F\left(\frac{x+\nu}{n}\right)=\sum_{d=1}^{\infty} \lambda_{n}(d) F(d x) \quad(n \in \mathbb{N})
$$

with complex valued sequences $\lambda_{n}$. This leads to new results on the periodic integrable and the aperiodic continuous solutions $F: \mathbb{R}_{+} \rightarrow \mathbb{C}$ interrelating the theory of functional equations and the theory of arithmetic functions.

1. Replicativity equations. The classical system of replicativity equations

$$
\frac{1}{n} \sum_{\nu=0}^{n-1} F\left(\frac{x+\nu}{n}\right)=n^{-s} F(x)
$$

for all $n \in \mathbb{N}$ with some fixed parameter $s \in \mathbb{C}$ was studied frequently. Usually $x$ varies over the additive group $\mathbb{T}=\mathbb{R} / \mathbb{Z}$ or a suitable interval $D \subseteq \mathbb{R}$, for instance $(0,1)$ or $\mathbb{R}_{+}$. For $D=\mathbb{Q} / \mathbb{Z}$ see Kubert [3]. The solutions $F$ of the above system form a linear space. Since any two equations $\left(1_{m}\right)$ and $\left(1_{n}\right)$ imply $\left(1_{m n}\right)$, it would suffice to assume the equations $\left(1_{n}\right)$ for all $n \in \mathbb{P}=\{p \in \mathbb{N}: p$ prime $\}$. The case $s=1$ was studied by Artin [1] in connection with the gamma function. He proved that every integrable solution $F: \mathbb{T} \rightarrow \mathbb{C}$ of the system $\left(1_{n}\right)$ is a linear combination of $\log \left(1-e^{2 \pi i x}\right)$ and its conjugate almost everywhere. For arbitrary $s \in \mathbb{C}$ Milnor [8] gave a complete

1991 Mathematics Subject Classification: 11A25, 39B62, 39A10. 
characterization of the space of continuous solutions $F:(0,1) \rightarrow \mathbb{C}$ and discussed their continuous extensions to $\mathbb{T}$ and to $\mathbb{R}_{+}$. In particular, this leads to linear relations between the polylogarithm and the Hurwitz zeta function, and there are significant applications to Dirichlet $L$-functions, the Riemann zeta function, the gamma function and related functions. Yoder [12] replaced the factors $n^{-s}$ on the right side of the equations $\left(1_{n}\right)$ by the values $g(n)$ of a generating sequence $g: \mathbb{N} \rightarrow \mathbb{C}$ and showed that the existence of non-trivial solutions $F$ forces $g$ to be totally multiplicative, which means $g(1)=1$ and $g(m n)=g(m) g(n)$ for all $m, n \in \mathbb{N}$. Moreover, the existence of aperiodic continuous solutions $F: \mathbb{R}_{+} \rightarrow \mathbb{C}$ implies $g(n)=n^{-s}$ with some $s \in \mathbb{C}$ and hence leads back to the classical system.

The aim of this paper is to investigate the system $\mathscr{S}$ of extended replicativity equations, previously introduced in [6],

$$
\frac{1}{n} \sum_{\nu=0}^{n-1} F\left(\frac{x+\nu}{n}\right)=\sum_{d=1}^{\infty} \lambda_{n}(d) F(d x)
$$

with some fixed sequences $\lambda_{n}: \mathbb{N} \rightarrow \mathbb{C}$ for all $n \in \mathbb{N}$. This system provides a natural extension of the classical replicativity system as it removes the restraint on the generating sequence $g$ to have the form $g(n)=n^{-s}$ or to be totally multiplicative at least. Namely, consider $F: \mathbb{T} \rightarrow \mathbb{C}$ as restriction of a function $G$, holomorphic on the open unit disc $U \subseteq \mathbb{C}$ and normalized by $G(0)=0$ and $G^{\prime}(0)=1$, to the boundary $\partial U$. Set

$$
G(z)=\sum_{a=1}^{\infty} g(a) z^{a}
$$

and write $F(x)=G(e(x))$, where $e(x)=e^{2 \pi i x}$ for $x \in \mathbb{R}$. The identity

$$
\frac{1}{n} \sum_{\nu=0}^{n-1} e\left(a \frac{\nu}{n}\right)= \begin{cases}1 & \text { if } n \mid a \\ 0 & \text { otherwise }\end{cases}
$$

yields

$$
\frac{1}{n} \sum_{\nu=0}^{n-1} G\left(z e\left(\frac{\nu}{n}\right)\right)=\sum_{\substack{a=1 \\ n \mid a}}^{\infty} g(a) z^{a} \quad(z \in U) .
$$

Now, apart from a remainder term of order $|z|^{(d+1) n}$, the right side can be written as a linear combination $\lambda_{n}(1) G\left(z^{n}\right)+\lambda_{n}(2) G\left(z^{2 n}\right)+\ldots+\lambda_{n}(d) G\left(z^{d n}\right)$ of $G\left(z^{n}\right), \ldots, G\left(z^{d n}\right)$ with uniquely determined coefficients $\lambda_{n}(1), \ldots, \lambda_{n}(d)$ $\in \mathbb{C}$ for every $d \in \mathbb{N}$. Assume that the remainder term vanishes as $d \rightarrow \infty$. Then

$$
\frac{1}{n} \sum_{\nu=0}^{n-1} G\left(z e\left(\frac{\nu}{n}\right)\right)=\sum_{d=1}^{\infty} \lambda_{n}(d) G\left(z^{n d}\right) \quad(z \in U)
$$


and in passing to $F$ with $x$ replaced by $x / n$ we formally arrive at $\left(2_{n}\right)$. Notice that $\mathscr{S}$ reduces to Yoder's system if and only if $g$ is totally multiplicative, in which case $\lambda_{n}(1)=g(n)$ and $\lambda_{n}(d)=0$ for all $d \in \mathbb{N}, d \neq 1$, and all $n \in \mathbb{N}$.

2. Periodic solutions. Let $L(\mathbb{T})$ denote the space of integrable functions $F: \mathbb{T} \rightarrow \mathbb{C}$ modulo null functions under the norm

$$
\|F\|=\int_{\mathbb{T}}|F(x)| d x<\infty .
$$

The above construction of the system $\mathscr{S}$ suggests investigating its solutions $F \in L(\mathbb{T})$. Evidently $\mathscr{S}$ has non-trivial constant solutions $F$ if and only if

$$
\sum_{d=1}^{\infty} \lambda_{n}(d)=1
$$

holds for all $n \in \mathbb{N}$. The existence of non-constant solutions $F \in L(\mathbb{T})$ depends on how the sequences $\lambda_{n}(n \in \mathbb{N})$ are interrelated, which requires some arithmetical preparation. Denote by $\mathcal{F}$ the complex algebra of arithmetical sequences $g: \mathbb{N} \rightarrow \mathbb{C}$ endowed with the usual pointwise linear operations and with the Dirichlet convolution $*: \mathcal{F}^{2} \rightarrow \mathcal{F}$ as multiplication, defined by

$$
(f * g)(m)=\sum_{\substack{a, b \in \mathbb{N} \\ a \cdot b=m}} f(a) g(b) \quad(m \in \mathbb{N}) .
$$

Observe that $\mathcal{F}$ contains the multiplicative identity $\varepsilon$ with $\varepsilon(1)=1$ and $\varepsilon(m)=0$ for all $m \in \mathbb{N}, m \neq 1$. The multiplicative group of $\mathcal{F}$ is $\mathcal{F}^{*}=$ $\{g \in \mathcal{F}: g(1) \neq 0\}$. By $g^{-1}$ we denote the multiplicative inverse of $g \in \mathcal{F}^{*}$, which means $g * g^{-1}=\varepsilon$. For $g \in \mathcal{F}$ and $n \in \mathbb{N}$ we define the subsequence $g(n \bullet) \in \mathcal{F}$ by $g(n \bullet)(a)=g(n a)$ for all $a \in \mathbb{N}$.

Theorem 1. For arbitrary sequences $\lambda_{n} \in \mathcal{F}(n \in \mathbb{N})$, let $g \in \mathcal{F}$ be defined by $g(n)=\lambda_{n}(1)$ for all $n \in \mathbb{N}$. If the system $\mathscr{S}$ has non-constant solutions $F \in L(\mathbb{T})$ then

(a) $g(1)=1$ and $\lambda_{n}=g^{-1} * g(n \bullet)$ for all $n \in \mathbb{N}$,

(b) the Fourier coefficients of $F$ are given by

$$
\widehat{F}(m)= \begin{cases}c_{\mathrm{sgn} m} g(|m|) & \text { if } m \in \mathbb{Z}^{\times}=\mathbb{Z} \backslash\{0\}, \\ c_{0} & \text { if } m=0,\end{cases}
$$

with parameters $c_{0}, c_{1}, c_{-1} \in \mathbb{C}$,

(c) $c_{0}=0$, unless $\left(3_{n}\right)$ is valid for all $n \in \mathbb{N}$.

Proof. Let

$$
\sum_{m \in \mathbb{Z}} \widehat{F}(m) e(m x) \quad(x \in \mathbb{T})
$$


be the Fourier series of $F \in L(\mathbb{T})$. The Fourier coefficients are given by

$$
\widehat{F}(m)=\int_{\mathbb{T}} F(x) e(-m x) d x \quad(m \in \mathbb{Z}) .
$$

Denoting the left side of $\left(2_{n}\right)$ by $F_{n}(x)$, we see that $F_{n} \in L(\mathbb{T})$ and $\widehat{F}_{n}(m)=$ $\widehat{F}(n m)$ for all $m \in \mathbb{Z}$. Denoting the right side of equation $\left(2_{n}\right)$ by $S_{n}(x)$, we obtain $S_{n} \in L(\mathbb{T})$ from the convergence of the series to $F_{n} \in L(\mathbb{T})$, which legalizes termwise integration and shows that

$$
\widehat{S}_{n}(m)=\sum_{d \mid m} \lambda_{n}(d) \widehat{F}(m / d) \quad(m \in \mathbb{Z}) .
$$

Comparing the coefficients leads to

$$
\widehat{F}(n m)=\sum_{d \mid m} \lambda_{n}(d) \widehat{F}(m / d) \quad(m \in \mathbb{Z}) .
$$

In particular, for $m= \pm 1$ this implies $\widehat{F}( \pm n)=g(n) \widehat{F}( \pm 1)$. Since, by assumption, $\widehat{F} \not \equiv 0$ on $\mathbb{Z}^{\times}$, we obtain $g(1)=1$ and $g(n m)=\left(\lambda_{n} * g\right)(m)$ for all $m \in \mathbb{N}$, which is equivalent to (a). Putting $c_{1}=\widehat{F}(1), c_{-1}=\widehat{F}(-1)$ and $c_{0}=\widehat{F}(0)$ we obtain (b), and (c) follows from (4) for $m=0$.

In the sequel we only consider systems $\mathscr{S}$ for which a sequence $g \in \mathcal{F}^{*}$ according to Theorem 1(a) exists. We call $g$ the generating sequence of $\mathscr{S}$. By Theorem 1 the dimension of the $\mathbb{C}$-linear space $P_{g}$ of solutions $F \in L(\mathbb{T})$ of $\mathscr{S}$ is at most 3 . In fact, for a large class of generating sequences we can reduce this bound by 1 .

For $q \in \mathbb{N}, q \neq 1$, denote by $\mathcal{R}_{q}$ the set of all sequences $g \in \mathcal{F}$ satisfying a recurrence equation of the form

$$
g\left(q^{l+k} \bullet\right)+c_{k-1} g\left(q^{l+k-1} \bullet\right)+\ldots+c_{0} g\left(q^{l} \bullet\right)=0
$$

with certain integers $k, l \geq 0$ and complex coefficients $c_{0} \neq 0, c_{1}, \ldots, c_{k-1}$. For every fixed $g \in \mathcal{R}_{q}$ there exist minimal numbers $k=k(q), l=l(q) \in$ $\mathbb{N}_{0}=\mathbb{N} \cup\{0\}$ such that $\left(5_{q}\right)$ holds. For this choice of $k, l$ we associate with $g \in \mathcal{R}_{q}$ the companion polynomial

$$
f_{q}(z)=z^{k}+c_{k-1} z^{k-1}+\ldots+c_{0} \in \mathbb{C}[z]
$$

and the set $Z_{q}=\left\{\zeta \in \mathbb{C}: f_{q}(\zeta)=0\right\}$ of its zeros. By $k_{\zeta} \in \mathbb{N}$ we denote the multiplicity of $\zeta \in Z_{q}$.

Lemma 1. Let $g \in \mathcal{R}_{q}$ for some $q>1$. Then, for every $a \in \mathbb{N}$, the solutions of the recurrence equation $\left(5_{q}\right)$ are given by

$$
g\left(q^{\nu} a\right)=\sum_{\zeta \in Z_{q}} P_{\zeta}(\nu) \zeta^{\nu} \quad\left(\nu \in \mathbb{N}_{0}, \nu \geq l\right)
$$


with polynomials $P_{\zeta}(x) \in \mathbb{C}[x]$, depending on $a \in \mathbb{N}$, of degree $\leq k_{\zeta}-1$ or $P_{\zeta}(x) \equiv 0$.

Proof. For fixed $a \in \mathbb{N}$ set $h(\nu)=g\left(q^{\nu} a\right)$. Then $\left(5_{q}\right)$ implies

$$
h(\nu+k)+c_{k-1} h(\nu+k-1)+\ldots+c_{0} h(\nu)=0 \quad(\nu \geq l) .
$$

As is well known (see, for instance, Lidl and Niederreiter [5], Chapter 6, or Methfessel [7], Section 1), the solutions $h \in \mathcal{F}$ of this recurrence equation have the form

$$
h(\nu)=\sum_{\zeta \in Z_{q}} P_{\zeta}(\nu) \zeta^{\nu}
$$

with certain polynomials $P_{\zeta}(x) \in \mathbb{C}[x]$, according to the statement of Lemma 1.

Theorem 2. Let $g \in \mathcal{R}_{q}$ for some $q>1$ and $g(1)=1$. Then the complex vector space $P_{g}$ has dimension $\leq 2$. If $\widehat{F}(0) \neq 0$ for some $F \in P_{g}$ then $P_{g}$ consists of all constant functions, and $\operatorname{dim} P_{g}=1$.

Proof. In view of Theorem 1 we only need to consider the case $\left(3_{n}\right)$ for all $n \in \mathbb{N}$. By taking the convolution product with $g^{-1}$ we may rewrite $\left(5_{q}\right)$ as

$$
\lambda_{q^{l+k}}+c_{k-1} \lambda_{q^{l+k-1}}+\ldots+c_{0} \lambda_{q^{l}}=0
$$

Summation over $d \in \mathbb{N}$ of the values at $d$ and insertion of $\left(3_{n}\right)$ for $n \in$ $\left\{q^{l}, \ldots, q^{l+k}\right\}$ leads to $f_{q}(1)=0$, which is equivalent to $1 \in Z_{q}$. Hence, by Lemma 1 and the minimality of $k=k(q)$,

$$
g\left(q^{\nu} a\right)=P_{1}(\nu)+\sum_{\zeta \in Z_{q} \backslash\{1\}} P_{\zeta}(\nu) \zeta^{\nu} \quad(\nu \geq l)
$$

with certain polynomials $P_{\zeta}(x) \in \mathbb{C}[x]$ depending on $a \in \mathbb{N}$, and $P_{1}(x) \not \equiv 0$ for some $a \in \mathbb{N}$. From this we conclude that $g(n) \neq \mathcal{O}(1)$ as $n \rightarrow \infty$. Finally, Theorem 1 and the Riemann-Lebesgue lemma yield $c_{1}=c_{-1}=0$.

As to the converse of Theorems 1 and 2, we add the following result.

THEOREM 3. Assume that the Fourier series of $F \in L(\mathbb{T})$ has the form

$$
\sum_{m \in \mathbb{Z}^{\times}} c_{\mathrm{sgn} m} g(|m|) e(m x)
$$

with some sequence $g \in \mathcal{F}, g(1)=1$, and some constants $c_{1}, c_{-1} \in \mathbb{C}$, and let the system $\mathscr{S}$ be generated by $g$. If all series

$$
\sum_{d=1}^{\infty} \lambda_{n}(d) F(d x) \quad(n \in \mathbb{N})
$$

converge in $L(\mathbb{T})$ then $F \in P_{g}$. 
Proof. Recall that, by Fejér's theorem, the series

$$
\sum_{m \in \mathbb{Z}^{\times}} c_{\operatorname{sgn} m} g(|m|) e(m x) \quad(x \in \mathbb{T})
$$

is Cesàro-summable to $F \in L(\mathbb{T})$. With the notation of the proof of Theorem $1, F_{n} \in L(\mathbb{T})$ has the Fourier series

$$
\sum_{m \in \mathbb{Z}^{\times}} c_{\mathrm{sgn} m} g(n|m|) e(m x) \quad(x \in \mathbb{T}) .
$$

By assumption, the series $S_{n}(x)$ converges for almost all $x \in \mathbb{T}$, and $S_{n} \in$ $L(\mathbb{T})$. A short calculation similar to that in the proof of Theorem 1 shows that

$$
\sum_{m \in \mathbb{Z}^{\times}} \sum_{d \mid m} \lambda_{n}(d) \widehat{F}(m / d) e(m x) \quad(x \in \mathbb{T})
$$

is the Fourier series of $S_{n}$. Now observe that

$$
\sum_{d \mid m} \lambda_{n}(d) \widehat{F}(m / d)=c_{\mathrm{sgn} m}\left(\lambda_{n} * g\right)(|m|)=c_{\mathrm{sgn} m} g(n|m|) \quad\left(m \in \mathbb{Z}^{\times}\right) .
$$

Hence the Fourier series of $F_{n}$ and $S_{n}$ coincide, which gives $F_{n}=S_{n}$ in $L(\mathbb{T})$, and the assertion of Theorem 3 follows.

3. Multiplicative sequences. A sequence $g: \mathbb{N} \rightarrow \mathbb{C}$ is called multiplicative if $g(1)=1$ and $g(m n)=g(m) g(n)$ for all coprime $m, n \in \mathbb{N}$. The set $\mathcal{M}$ of multiplicative sequences forms a subgroup of $\mathcal{F}^{*}$ under the Dirichlet convolution. By $\mathcal{T}$ we denote the subset of totally multiplicative sequences, and we write $\langle n\rangle$ and $\langle T\rangle$ for the set of all $d \in \mathbb{N}$ whose prime factors divide $n \in \mathbb{N}$, and for the multiplicative semigroup generated by the set $T \subseteq \mathbb{P}$ respectively. First, we restate Theorem 2 from [6] as

Lemma 2. Let $g \in \mathcal{F}, g(1)=1$, and $\lambda_{n}=g^{-1} * g(n \bullet)$ for all $n \in \mathbb{N}$. Then $g \in \mathcal{M}$ is equivalent to $\operatorname{supp} \lambda_{n} \subseteq\langle n\rangle$ for all $n \in \mathbb{N}$, and in this case $\lambda_{m n}=\lambda_{m} * \lambda_{n}$ for all coprime $m, n \in \mathbb{N}$.

By Lemma 2, every system $\mathscr{S}$ generated by some $g \in \mathcal{M}$ can equivalently be replaced by the partial system of equations $\left(2_{n}\right)$ for all $n \in \mathbb{P}^{*}=\left\{p^{\nu}\right.$ : $p \in \mathbb{P}, \nu \in \mathbb{N}\}$.

With $g \in \mathcal{M}$ and $p \in \mathbb{P}$ we associate the formal Dirichlet series

$$
\widetilde{g}_{p}(s)=\sum_{k=0}^{\infty} g\left(p^{k}\right) p^{-k s} \quad(s \in \mathbb{C}),
$$

and we denote by $\mathcal{M}_{p}$ the set of all $g \in \mathcal{M}$ having the property that $\widetilde{g}_{p}(s)$ is absolutely convergent and zero-free in the closed half plane $\operatorname{Re} s \geq 0$. The next theorem extends Theorem 5 in [6]. 
TheORem 4. Let $g \in \mathcal{M}_{p}$ for some $p \in \mathbb{P}$. Then the complex vector space $P_{g}$ has dimension $\leq 2$. In particular, $\widehat{F}(0)=0$ for every $F \in P_{g}$.

Proof. It follows from Wiener's inversion theorem (see, for instance, Rudin [11], Chapter 18) applied to the power series $G_{p}(z)=\widetilde{g}_{p}(s)$ with $z=p^{-s}$ that $\mathcal{M}_{p}$ forms a subgroup of $\mathcal{M}$, for every $p \in \mathbb{P}$. For $n=p^{\nu} \in \mathbb{P}^{*}$ we infer from Lemma 2 that

$$
\begin{aligned}
\sum_{d=1}^{\infty}\left|\lambda_{n}(d)\right| & =\sum_{k=0}^{\infty}\left|\lambda_{n}\left(p^{k}\right)\right| \\
& \leq \sum_{\varrho=0}^{\infty}\left|g^{-1}\left(p^{\varrho}\right)\right| \sum_{\sigma=\nu}^{\infty}\left|g\left(p^{\sigma}\right)\right| \ll \sum_{\sigma=\nu}^{\infty}\left|g\left(p^{\sigma}\right)\right|=\mathcal{O}(1)
\end{aligned}
$$

as $\nu \rightarrow \infty$. Hence equation $\left(3_{n}\right)$ fails to hold for all $n \in \mathbb{N}$, and Theorem 1 gives the desired conclusion.

A local version of Theorem 4 is easily obtained by assuming $g(1)=1$ and $\operatorname{supp} \lambda_{n} \subseteq\langle p\rangle$ for all $n \in\langle p\rangle$ instead of the global multiplicativity of the sequence $g$ in the definition of $\mathcal{M}_{p}$.

The proof of Theorem 4, similar to that of Theorem 2, consists in excluding certain generating sequences $g$ of $\mathscr{S}$ satisfying the equations $\left(3_{n}\right)$, $n \in \mathbb{N}$. It would therefore be desirable to know how to reconstruct $g \in \mathcal{M}$ from an infinite system of equations of the type

$$
f(n)=\sum_{d=1}^{\infty} \lambda_{n}(d) h(d)
$$

for all $n \in \mathbb{N}$, where $h \in \mathcal{T}$ and $f \in \mathcal{F}$ are suitably chosen sequences.

Lemma 3. For $g \in \mathcal{F}$ with $g(1)=1$ let $\lambda_{n}=g^{-1} * g(n \bullet)$ for all $n \in \mathbb{N}$, and let $h \in \mathcal{T}$. Suppose that all series $\left(7_{n}\right)$ for $n \in \mathbb{N}$ are convergent, so that $f \in \mathcal{F}$ is defined and $f(1)=1$. If $g \in \mathcal{M}$ then $f \in \mathcal{M}$, and if $g \in \mathcal{T}$ then $f \in \mathcal{T}$. Conversely, if $f \in \mathcal{T}$ and $f(p) h(p) \neq 1$ for all $p \in \mathbb{P}$ then there exists a uniquely determined $g \in \mathcal{M}$ such that $\left(7_{n}\right)$ is valid for all $n \in \mathbb{N}$, namely $g=f \in \mathcal{T}$. This implication does not hold for $f \in \mathcal{T}$ if $f(p) h(p)=1$ for some $p \in \mathbb{P}$.

Proof. The Dirichlet series

$$
\sum_{d=1}^{\infty} \frac{\lambda_{n}(d) h(d)}{d^{s}} \quad(n \in \mathbb{N})
$$

converges for $s=0$ and hence for $\operatorname{Re} s>0$. The convergence is absolute for Re $s>1$ so that, for $g \in \mathcal{M}$ and coprime $m, n \in \mathbb{N}$, Lemma 2 yields the 
equation

$$
\sum_{d=1}^{\infty} \frac{\lambda_{m n}(d) h(d)}{d^{s}}=\sum_{a=1}^{\infty} \frac{\lambda_{m}(a) h(a)}{a^{s}} \cdot \sum_{b=1}^{\infty} \frac{\lambda_{n}(b) h(b)}{b^{s}},
$$

which extends analytically to the half plane $\operatorname{Re} s>0$. By letting $s \rightarrow 0$ along the positive real axis, Abel's limit theorem for Dirichlet series gives $f(m n)=f(m) f(n)$, i.e. $f \in \mathcal{M}$. If, moreover, $g \in \mathcal{T}$ then $\lambda_{n}=g(n) \varepsilon$ for all $n \in \mathbb{N}$, and $f=g \in \mathcal{T}$ follows.

Next, Lemma 2 implies the identity

$$
\lambda_{p^{\nu}}\left(p^{k}\right)-\lambda_{p^{\nu+1}}\left(p^{k-1}\right)=g\left(p^{\nu}\right) g^{-1}\left(p^{k}\right)
$$

valid for $g \in \mathcal{M}, p \in \mathbb{P}, \nu \in \mathbb{N}_{0}, k \in \mathbb{N}$. Multiply with $h\left(p^{k}\right)$ and sum over $k \in \mathbb{N}$ to obtain

$$
f\left(p^{\nu}\right)-h(p) f\left(p^{\nu+1}\right)=g\left(p^{\nu}\right) \sum_{k=0}^{\infty} g^{-1}\left(p^{k}\right) h\left(p^{k}\right) \quad\left(p \in \mathbb{P}, \nu \in \mathbb{N}_{0}\right) .
$$

In particular, for $\nu=0$,

$$
1-h(p) f(p)=\sum_{k=0}^{\infty} g^{-1}\left(p^{k}\right) h\left(p^{k}\right) \quad(p \in \mathbb{P}) .
$$

By inserting (10) into (9) and dividing by $1-h(p) f(p) \neq 0$ we see that $f \in \mathcal{T}$ implies $g\left(p^{\nu}\right)=f\left(p^{\nu}\right)$ for all $p^{\nu} \in \mathbb{P}^{*}$ and hence $g=f$. The last assertion of Lemma 2 results from the following construction.

ExAmple 1. Let $f, h \in \mathcal{T}, T=\{p \in \mathbb{P}: f(p) h(p)=1\} \neq \emptyset$ and $q: T \rightarrow \mathbb{N}$ such that $q(p) \geq p$ for $p \in T$. Then $g \in \mathcal{M}$ defined by

$$
g\left(p^{k}\right)= \begin{cases}f\left(p^{k}\right)(-1)^{k}\left(\begin{array}{c}
-1 / q(p) \\
k
\end{array}\right) & \text { if } p \in T, \\
f\left(p^{k}\right) & \text { if } p \notin T,\end{cases}
$$

satisfies $\left(7_{n}\right)$ for all $n \in \mathbb{N}$ and, moreover, $g(n)=\mathcal{O}(|f(n)|)$ as $n \rightarrow \infty$, $n \in\langle T\rangle$.

In order to verify the assertions we may restrict our attention to $n \in\langle T\rangle$. First, observe that $(g h)^{-1} *(g h)(n \bullet)=\lambda_{n} h(n \bullet)$, and replace $g h$ by $g$ and $f h$ by $f$. Then it suffices to show that the restriction of the sequence $g \in \mathcal{M}$ with

$$
g\left(p^{k}\right)=(-1)^{k}\left(\begin{array}{c}
-1 / q(p) \\
k
\end{array}\right) \quad(p \in T, k \in \mathbb{N})
$$

to $\langle T\rangle$ satisfies both $g\left(p^{k}\right)=\mathcal{O}(1)$ as $p^{k} \rightarrow \infty$, and $\left(3_{n}\right)$ for all $n \in\langle T\rangle$. 
In fact, we have the estimate

$$
\begin{aligned}
0<g\left(p^{k}\right) & =\frac{1}{k q(p)} \prod_{\kappa=1}^{k-1}\left(1+\frac{1}{\kappa q(p)}\right) \leq \frac{1}{k q(p)}\left(\prod_{\varrho=0}^{(k-1) q(p)}\left(1+\frac{1}{\varrho}\right)\right)^{1 / q(p)} \\
& =\frac{1}{k q(p)}((k-1) q(p)+1)^{1 / q(p)}<(k q(p))^{-1+1 / q(p)}
\end{aligned}
$$

which gives the $\mathcal{O}$-relation. Next, consider for $|z|<1$ the power series

$$
G_{p}(z)=\sum_{k=0}^{\infty} g\left(p^{k}\right) z^{k}=(1-z)^{-1 / q(p)} \neq 0,
$$

and invert to obtain

$$
G_{p}^{-1}(z)=\sum_{k=0}^{\infty} g^{-1}\left(p^{k}\right) z^{k}=(1-z)^{1 / q(p)}=\sum_{k=0}^{\infty}(-1)^{k}\left(\begin{array}{c}
1 / q(p) \\
k
\end{array}\right) z^{k}
$$

Hence

$$
g^{-1}\left(p^{k}\right)=(-1)^{k}\left(\begin{array}{c}
1 / q(p) \\
k
\end{array}\right) \quad(p \in T, k \in \mathbb{N}),
$$

and, similar to the above,

$$
0<-g^{-1}\left(p^{k}\right) \leq \frac{1}{q(p)} k^{-1-1 / q(p)} \quad(p \in T, k \in \mathbb{N}) .
$$

This shows the absolute convergence of the power series $G_{p}^{-1}(z)$ even for $|z| \leq 1$, and Abel's limit theorem for power series yields

$$
\sum_{k=0}^{\infty} g^{-1}\left(p^{k}\right)=0 \quad(p \in T)
$$

Finally, for $|z|<1$ and $n=p^{\nu}$ with $p \in T, \nu \in \mathbb{N}$ we find the representation

$$
\sum_{k=0}^{\infty} \lambda_{n}\left(p^{k}\right) z^{k}=G_{p}^{-1}(z) \sum_{k=0}^{\infty} g\left(p^{\nu+k}\right) z^{k}=z^{-\nu}\left(1-G_{p}^{-1}(z) \sum_{k=0}^{\nu-1} g\left(p^{k}\right) z^{k}\right),
$$

the right side of which is an absolutely convergent power series for $|z| \leq 1$. By applying Abel's limit theorem again, we arrive at $\left(3_{n}\right)$ for all $n \in T$.

In particular, Example 1 shows that there exist infinitely many sequences $g \in \mathcal{M}$ satisfying $\left(3_{n}\right)$ for all $n \in \mathbb{N}$, with $g(n)=\mathcal{O}(1)$ as $n \rightarrow \infty$. We leave the problem open whether the corresponding series

$$
\sum_{n=1}^{\infty} g(n) e(n x) \quad(x \in \mathbb{T})
$$

can be the Fourier series of $L(\mathbb{T})$ functions. 
4. Multiplicative recurrent sequences. According to Theorem 1(a), every sequence $g \in \mathcal{F}$ with $g(1)=1$ uniquely determines a system $\mathscr{S}$ of functional equations. Apart from the classical case $g \in \mathcal{T}$, where $\lambda_{n}=g(n) \varepsilon$ for all $n \in \mathbb{N}$, there exists no general explicit formula for the corresponding sequences $\lambda_{n} \in \mathcal{F}$. However, for generating sequences $g \in \mathcal{M} \cap \mathcal{R}_{q}$ we describe a constructive method based on Lemmas 1 and 2, which enables us to determine $\mathscr{S}$ in many cases. The following theorem characterizes the local polynomial-exponential type structure of sequences $g \in \mathcal{M} \cap \mathcal{R}_{q}$.

TheOREM 5. For $g \in \mathcal{M}$ the following assertions are equivalent:

(a) $g \in \mathcal{R}_{p}$ for some $p \in \mathbb{P}$,

(b) $g \in \mathcal{R}_{q}$ for some $q \in \mathbb{N}, q \neq 1$,

(c) there exist integers $p \in \mathbb{P}, k, l \in \mathbb{N}_{0}$ with $k+l \neq 0$, and coefficients $c_{0}, \ldots, c_{k-1} \in \mathbb{C}, c_{0} \neq 0$, such that $g$ satisfies the linear recurrence equation

$$
g\left(p^{\nu+k}\right)+c_{k-1} g\left(p^{\nu+k-1}\right)+\ldots+c_{0} g\left(p^{\nu}\right)=0 \quad\left(\nu \in \mathbb{N}_{0}, \nu \geq l\right),
$$

(d) there exist integers $p \in \mathbb{P}, k, l, r \in \mathbb{N}_{0}$ with $k+l \neq 0, k_{1}, \ldots, k_{r} \in \mathbb{N}$ satisfying $k_{1}+\ldots+k_{r}=k$, and polynomials $P_{\varrho}(x) \in \mathbb{C}[x]$ of degree $\leq k_{\varrho}-1$ or $P_{\varrho}(x) \equiv 0(\varrho=1, \ldots, r)$, such that

$$
g\left(p^{\nu}\right)=\sum_{1 \leq \varrho \leq r} P_{\varrho}(\nu) \zeta_{\varrho}^{\nu} \quad\left(\nu \in \mathbb{N}_{0}, \nu \geq l\right) .
$$

Pr o of. Trivially, (a) implies (b). If $g \in \mathcal{R}_{q}$ and $q \neq 1$ is a power of $p \in \mathbb{P}$ then a recurrence equation of type (c) follows from (b) by taking $a=p^{\nu}$ with $\nu \in \mathbb{N}_{0}$ in $\left(5_{q}\right)$. Now, let $q=q_{1} q_{2}$ be any coprime decomposition with $q_{1}, q_{2} \in \mathbb{N} \backslash\{1\}$ and a prime power $q_{2}$. If $g\left(q_{1}^{\varrho} b\right) \neq 0$ for infinitely many exponents $\varrho \in \mathbb{N}$ and some $b \in \mathbb{N}$ with $\left(b, q_{2}\right)=1$ then it follows from (b) and $g \in \mathcal{M}$ by taking $a=q_{1}^{\varrho} q_{2}^{\nu} b$ in $\left(5_{q}\right)$ that

$$
\begin{aligned}
g\left(q_{1}^{l+\varrho+k} b\right) g\left(q_{2}^{l+\nu+k}\right)+c_{k-1} g\left(q_{1}^{l+\varrho+k-1} b\right) g\left(q_{2}^{l+\nu+k-1}\right)+\ldots \\
+c_{0} g\left(q_{1}^{l+\varrho} b\right) g\left(q_{2}^{l+\nu}\right)=0 .
\end{aligned}
$$

Fix $\varrho$ and $b$ such that $g\left(q_{1}^{l+\varrho} b\right) \neq 0$. Then

$$
d_{k} g\left(q_{2}^{l+\nu+k}\right)+d_{k-1} g\left(q_{2}^{l+\nu+k-1}\right)+\ldots+d_{0} g\left(q_{2}^{l+\nu}\right)=0
$$

with certain coefficients $d_{0}, \ldots, d_{k-1}, d_{k} \in \mathbb{C}, d_{0} \neq 0$, which gives a recurrence equation according to (c). If $g\left(q_{1}^{\varrho} b\right)=0$ for all sufficiently large $\varrho \in \mathbb{N}$ and all $b \in \mathbb{N}$ with $\left(b, q_{2}\right)=1$ then, by the multiplicativity of $g$, also $g\left(q_{1}^{\varrho} a\right)=0$ for all sufficiently large $\varrho \in \mathbb{N}$ and all $a \in \mathbb{N}$. Hence $g \in \mathcal{R}_{q_{1}}$ where $q_{1}$ has a reduced number of prime divisors. In this case proceed inductively to obtain (c).

The equivalence of (c) and (d) follows from Lemma 1. It remains to show that (c) implies (a). Every $a \in \mathbb{N}$ has a coprime decomposition of the 
form $a=p^{\nu-l} b$ with $\nu \in \mathbb{N}_{0}, \nu \geq l$. Now $g \in \mathcal{M}$ implies

$$
\begin{aligned}
g\left(p^{l+k} a\right)+c_{k-1} g\left(p^{l+k-1} a\right)+\ldots+c_{0} g\left(p^{l} a\right) & \\
& =g(b) \cdot\left(g\left(p^{\nu+k}\right)+c_{k-1} g\left(p^{\nu+k-1}\right)+\ldots+c_{0} g\left(p^{\nu}\right)\right)=0,
\end{aligned}
$$

which completes the proof.

With $g \in \mathcal{M}$ and $p \in \mathbb{P}$ we associate the $p$-fibre $g_{p} \in \mathcal{M}$ defined by

$$
g_{p}(n)=\left\{\begin{array}{ll}
g(n) & \text { if } n \in\langle p\rangle, \\
0 & \text { otherwise },
\end{array} \quad(n \in \mathbb{N})\right.
$$

and the formal power series

$$
G_{p}(z)=\sum_{\nu=0}^{\infty} g\left(p^{\nu}\right) z^{\nu} \quad(z \in \mathbb{C}),
$$

which takes the form of the Dirichlet series $\widetilde{g}_{p}(s)$ of $g_{p}$ by substituting $z=$ $p^{-s}$. Further we set $\mathcal{N}_{p}=\{g \in \mathcal{M}: g=\varepsilon$ on $\langle p\rangle\}$. Obviously $\mathcal{N}_{p} \subseteq \mathcal{R}_{p}$. The following theorem describes the multiplicative structure of $\mathcal{M} \cap \mathcal{R}_{p}$ with respect to the Dirichlet convolution.

TheOREM 6. For every $p \in \mathbb{P}, \mathcal{M} \cap \mathcal{R}_{p}$ forms a group under the Dirichlet convolution, and there is a group homomorphism $\psi$ with kernel $\mathcal{N}_{p}$ from $\mathcal{M} \cap \mathcal{R}_{p}$ onto the multiplicative group of all quotients $P(z) / Q(z)$ of relatively prime polynomials $P(z), Q(z) \in \mathbb{C}[z]$ satisfying $P(0)=Q(0)=1$. In particular,

$$
\psi(g)=G_{p}(z), \quad Q(z)=z^{k} f_{p}\left(\frac{1}{z}\right), \quad P(z)=Q(z) G_{p}(z),
$$

where $k \in \mathbb{N}_{0}$ is the degree of the companion polynomial $f_{p}(z)$ of $g \in \mathcal{M} \cap \mathcal{R}_{p}$.

P r o of. First, observe that for $g \in \mathcal{M}$ the rationality of the power series $G_{p}(z)$ is equivalent to $g \in \mathcal{R}_{p}$. Hence, if $g, h \in \mathcal{M} \cap \mathcal{R}_{p}$ then $g * h \in \mathcal{M}$, and the corresponding power series $G_{p}(z), H_{p}(z)$ as well as their Cauchy product

$$
G_{p}(z) H_{p}(z)=\sum_{\nu=0}^{\infty} \sum_{\varrho+\sigma=\nu} g_{p}\left(p^{\varrho}\right) h_{p}\left(p^{\sigma}\right) z^{\nu}
$$

are rational functions. This gives $g * h \in \mathcal{M} \cap \mathcal{R}_{p}$ and shows that $\mathcal{M} \cap \mathcal{R}_{p}$ is closed under the Dirichlet convolution.

Further, the inverse $g^{-1}$ of $g \in \mathcal{M}$ belongs to $\mathcal{M}$. From Theorem 5 we know that the power series $G_{p}(z)$, associated with $g \in \mathcal{M} \cap \mathcal{R}_{p}$ and representing a rational function, has positive radius of convergence, and $G_{p}(0)=1$. By the Cauchy-Taylor theorem, the same is true for $G_{p}^{-1}(z)$, and the Cauchy product with $G_{p}(z)$ obviously corresponds to $g^{-1} * g=\varepsilon$ on the set $\langle p\rangle$. Therefore, $g^{-1} \in \mathcal{M} \cap \mathcal{R}_{p}$. 
Next, notice that $\mathcal{N}_{p}$ is a subgroup of the group $\mathcal{M} \cap \mathcal{R}_{p}$. The mapping $g \mapsto g_{p}$ is clearly a group homomorphism with kernel $\mathcal{N}_{p}$. The Dirichlet series $\widetilde{g}_{p}(s)$ of $g_{p} \in \mathcal{M} \cap \mathcal{R}_{p}$ is absolutely convergent in some right half plane of the complex plane, and $g_{p} \mapsto G_{p}\left(p^{-s}\right)=\widetilde{g}_{p}(s)$ represents an isomorphic mapping from $\left(\mathcal{M} \cap \mathcal{R}_{p}\right) / \mathcal{N}_{p}$ onto the multiplicative group of rational power series $R(z)$ with a positive radius of convergence and $R(0)=1$.

Finally, by multiplying the recurrence equation in Theorem 5(c) with $z^{\nu+k}$ and summing over all $\nu \in \mathbb{N}_{0}$, we see that $G_{p}(z) z^{k} f_{p}(1 / z)$ is a polynomial $P(z)$ of degree $\leq k+l-1$ with $P(0)=1$, which is relatively prime to $Q(z)=z^{k} f_{p}(1 / z)$, due to the minimality of $k=k(p)$. This completes the proof of Theorem 6 .

The divisor function $\tau \in \mathcal{M}$ is defined by $\tau=1 * 1$, where 1 denotes the constant sequence with value 1 on $\mathbb{N}$.

Example 2. For $s \in \mathbb{C}$ let the system $\mathscr{S}$ be generated by the sequence $g=\left(\tau(n) n^{-s}\right) \in \mathcal{M}$. Observe that $g \in \mathcal{R}_{p}$ for every $p \in \mathbb{P}$ with $f_{p}(z)=$ $\left(z-p^{-s}\right)^{2}$. For $n=p^{\nu} \in \mathbb{P}^{*}$ we obtain

$$
\begin{aligned}
\sum_{k=0}^{\infty} \lambda_{n}\left(p^{k}\right) z^{k} & =\left(1-p^{-s} z\right)^{2} \sum_{\varrho=0}^{\infty}(\nu+\varrho+1) p^{-(\nu+\varrho) s} z^{\varrho} \\
& =p^{-\nu s}\left(\nu+1-\nu p^{-s} z\right),
\end{aligned}
$$

which gives

$$
\lambda_{p^{\nu}}\left(p^{k}\right)= \begin{cases}(\nu+1) p^{-\nu s} & \text { if } k=0 \\ -\nu p^{-(\nu+1) s} & \text { if } k=1 \\ 0 & \text { if } k>1\end{cases}
$$

Hence $\mathscr{S}$ consists of the equations

$$
\frac{1}{n} \sum_{\nu=0}^{n-1} F\left(\frac{x+\nu}{n}\right)=n^{-s} \sum_{d \mid n} \mu(d) \tau\left(\frac{n}{d}\right) d^{-s} F(d x) \quad(n \in \mathbb{N}),
$$

and $\operatorname{dim} P_{g} \leq 2$. In particular, for $\operatorname{Re} s \leq 0, s \neq 0$, the null function is the only solution $F \in L(\mathbb{T})$ of $\mathscr{S}$, and for $s=0$ the space $P_{g}$ of solutions $F \in L(\mathbb{T})$ of $\mathscr{S}$ consists of all constant functions. For Re $s>0$ that space is spanned by the cosine and the sine part of the series

$$
T(x)=\sum_{n=1}^{\infty} \tau(n) n^{-s} e^{2 \pi i n x},
$$

if these are the Fourier series of $L(\mathbb{T})$ functions respectively. This is obvious for Re $s>1$ only, due to the absolute convergence of $T(x)$.

In a similar way extended replicativity systems $\mathscr{S}$ generated, for instance, by the sequences $\left(\varphi(n) n^{-s}\right)$ or $\left(\sigma(n) n^{-s}\right)$, where $\varphi$ and $\sigma$ denote 
respectively the Euler function and the sum of divisors function, and their solutions $F \in L(\mathbb{T})$ can be treated.

5. Aperiodic solutions. For intervals $I \subseteq \mathbb{R}$ we denote by $C(I)$ the set of continuous functions $F: I \rightarrow \mathbb{C}$. We are interested in aperiodic solutions $F \in C\left(\mathbb{R}_{+}\right)$of systems $\mathscr{S}$, generated by some sequence $g \in \mathcal{F}, g(1)=1$. One may expect that they are rather exceptional.

It is well known that the Hurwitz zeta function defined by

$$
\zeta(s, x)=\sum_{\nu=0}^{\infty}(x+\nu)^{-s} \quad\left(\operatorname{Re} s>1, x \in \mathbb{R}_{+}\right)
$$

extends to a function holomorphic for $s \in \mathbb{C} \backslash\{1\}$ and $x \in \mathbb{C}_{\pi}=\mathbb{C} \backslash\{x \in$ $\mathbb{R}: x \leq 0\}$, with a simple pole at $s=1$ with residue 1 . The polylogarithm function defined by

$$
L_{s}(z)=\sum_{\nu=1}^{\infty} \nu^{-s} z^{\nu} \quad(s \in \mathbb{C}, z \in U)
$$

extends to a function holomorphic for $s \in \mathbb{C}$ and $z \in \mathbb{C} \backslash[1, \infty)$. In particular, we have the following two lemmas, which are taken from Milnor [8], Lemma 1, Lemma 2 and Theorem 1.

LEMMA 4. The function

$$
B_{s}(x)=-s \zeta(1-s, x)
$$

is holomorphic for $s \in \mathbb{C}$ and $x \in \mathbb{C}_{\pi}$, with $B_{0}(x)=1$. For each $s \in \mathbb{C}$ the function $B_{s}: \mathbb{R}_{+} \rightarrow \mathbb{C}$ solves both the classical replicativity system $\mathscr{S}$ generated by $g(n)=n^{-s}$ and the difference equation

$$
B_{s}(x+1)-B_{s}(x)=s x^{s-1} .
$$

Moreover, if $s \in \mathbb{N}_{0}$ then $B_{s}(x) \in \mathbb{Q}[x]$ is the sth Bernoulli polynomial and $B_{s}=B_{s}(0)$ is the sth Bernoulli number.

Lemma 5. For every $s \in \mathbb{C}$ the linear space consisting of all continuous solutions $F:(0,1) \rightarrow \mathbb{C}$ of the classical system $\mathscr{S}$ generated by $g(n)=n^{-s}$ has dimension 2. For $s \notin \mathbb{N}_{0}$ it is spanned by $B_{s}(x)$ and $B_{s}(1-x)$, for $s=0$ by $B_{0}(x)$ and $\cot (\pi x)$, for $s \in \mathbb{N}$ by $B_{s}(x)$ and $\operatorname{Re} L_{s}\left(e^{2 \pi i x}\right)$.

Now we can formulate our main result on aperiodic solutions of $\mathscr{S}$.

Theorem 7. Let $g \in \mathcal{M} \cap \mathcal{R}_{q}$ for two different $q \in \mathbb{P}$, and let the system $\mathscr{S}$ be generated by $g$. Then $\mathscr{S}$ has an aperiodic solution $F \in C\left(\mathbb{R}_{+}\right)$if and only if $\mathscr{S}$ is the classical system generated by $g(n)=n^{-s}$ with some $s \neq 0$. In this case

$$
F(x)=c B_{s}(x)+\Phi(x) \quad\left(x \in \mathbb{R}_{+}\right)
$$

with some constant $c \in \mathbb{C}^{\times}$and some 1 -periodic solution $\Phi \in C\left(\mathbb{R}_{+}\right)$of $\mathscr{S}$. 
The important point to note here is that the existence of aperiodic solutions $F \in C\left(\mathbb{R}_{+}\right)$of extended replicativity systems $\mathscr{S}$ generated by multiplicative recurrent sequences shrinks $\mathscr{S}$ to the classical system. This excludes the occurrence of aperiodic solutions $F \in C\left(\mathbb{R}_{+}\right)$in many cases. Observe that the previous result of Yoder [12], Theorem 4, is contained in Theorem 7 above, since every $g \in \mathcal{T}$ has the property $g \in \mathcal{M} \cap \mathcal{R}_{q}$ for all $q \in \mathbb{P}$.

Proof (of Theorem 7). By Lemmas 4 and 5 we only need to show that the existence of an aperiodic solution $F \in C\left(\mathbb{R}_{+}\right)$of $\mathscr{S}$ implies that $g(n)=n^{-s}$ with some $s \in \mathbb{C}$. We set

$$
\Delta(x)=F(x+1)-F(x) \quad\left(x \in \mathbb{R}_{+}\right)
$$

and denote for $\omega \in \mathbb{R}_{+}$by $R_{\omega}$ the ring of $\omega$-periodic functions $\eta \in C(\mathbb{R})$. The first step of the proof consists in deriving from $g \in \mathcal{R}_{q}$ for some $q \in \mathbb{N}$, $q \neq 1$, a functional equation of the type

$$
q^{-k} \Delta\left(\frac{x}{q^{k}}\right)+c_{k-1} q^{-(k-1)} \Delta\left(\frac{x}{q^{k-1}}\right)+\ldots+c_{0} \Delta(x)=0 \quad\left(x \in \mathbb{R}_{+}\right)
$$

with certain coefficients $c_{k-1}, \ldots, c_{0} \in \mathbb{C}, c_{0} \neq 0$, and from $g \in \mathcal{M}$ the system of functional equations

$$
\begin{aligned}
\Delta\left(\frac{x}{p^{\nu+1}}\right) & -p^{\nu} g\left(p^{\nu}\right) \Delta\left(\frac{x}{p}\right) \\
= & \frac{1}{p} \sum_{\varrho=0}^{p-1}\left(\Delta\left(\frac{x+\varrho}{p^{\nu+1}}\right)-p^{\nu} g\left(p^{\nu}\right) \Delta\left(\frac{x+\varrho}{p}\right)\right) \quad\left(x \in \mathbb{R}_{+}\right)
\end{aligned}
$$

for all $p \in \mathbb{P}$ and $\nu \in \mathbb{N}$. In the second step of the proof, while assuming (12) for two numbers $q \in \mathbb{N}$ having $\mathbb{Q}$-linearly independent logarithms (e.g. for different primes), we obtain the explicit formula

$$
\Delta(x)=\sum_{s \in S} P_{s}(\log x) x^{s-1} \quad\left(x \in \mathbb{R}_{+}\right)
$$

with some finite set $S \subseteq \mathbb{C}$ and certain polynomials $P_{s}(x) \in \mathbb{C}[x]$. Further we put (13) into the form

$$
\Delta\left(\frac{x}{p^{\nu+1}}\right)-p^{\nu} g\left(p^{\nu}\right) \Delta\left(\frac{x}{p}\right)=\sum_{\zeta \in T_{p}} h_{\zeta, p, \nu}(x) \zeta^{x},
$$

where $T_{p}$ is the set of zeros of the polynomial $t_{p}(z)=\left(z^{p}-1\right) /(z-1)-p$ and $h_{\zeta, p, \nu} \in R_{1}$. In the third step we insert (14) into (15) to conclude from $\Delta \not \equiv 0$ that $\Delta(x)=a x^{s-1}$ with some constants $a, s \in \mathbb{C}, a \neq 0$. Finally, we derive that $g(n)=n^{-s}$ for all $n \in \mathbb{N}$.

In particular, suppose that the system $\mathscr{S}$ is generated by some sequence $g \in \mathcal{R}_{q}, g(1)=1$, where $1 \neq q \in \mathbb{N}$. Then, similar to the proof of Theorem 2 , 
equation $\left(5_{q}\right)$ may be rewritten as

$$
\lambda_{q^{l+k}}+c_{k-1} \lambda_{q^{l+k-1}}+\ldots+c_{0} \lambda_{q^{l}}=0,
$$

from which it follows that

$$
\lambda_{q^{l+k}}(d) F(d x)+c_{k-1} \lambda_{q^{l+k-1}}(d) F(d x)+\ldots+c_{0} \lambda_{q^{l}}(d) F(d x)=0
$$

for all $d \in \mathbb{N}, x \in \mathbb{R}_{+}$. Summing over $d \in \mathbb{N}$ and writing again $F_{n}(x)$ for the left side of equation $\left(2_{n}\right)$, we infer that

$$
F_{q^{l+k}}(x)+c_{k-1} F_{q^{l+k-1}}(x)+\ldots+c_{0} F_{q^{l}}(x)=0 \quad\left(x \in \mathbb{R}_{+}\right) .
$$

Observe that $F_{n}(x+1)-F_{n}(x)=(1 / n) \Delta(x / n)$ for all $n \in \mathbb{N}$ and $x \in \mathbb{R}_{+}$, and subtract equation (16) at $x$ from that at $x+1$. It follows that

$\frac{1}{q^{l+k}} \Delta\left(\frac{x}{q^{l+k}}\right)+c_{k-1} \frac{1}{q^{l+k-1}} \Delta\left(\frac{x}{q^{l+k-1}}\right)+\ldots+c_{0} \frac{1}{q^{l}} \Delta\left(\frac{x}{q^{l}}\right)=0 \quad\left(x \in \mathbb{R}_{+}\right)$,

which is equivalent to (12).

Next, assume that the system $\mathscr{S}$ is generated by some sequence $g \in \mathcal{M}$. Observe that $\mathscr{S}$ turns into the system of functional equations for $\Delta$,

$$
\frac{1}{n} \Delta\left(\frac{x}{n}\right)=\sum_{d=1}^{\infty} \lambda_{n}(d) \sum_{\delta=0}^{d-1} \Delta(d x+\delta) \quad(n \in \mathbb{N}) .
$$

By multiplying the identity (8) with

$$
\sum_{\delta=0}^{p^{k}-1} \Delta\left(p^{k} x+\delta\right)
$$

summing over $k \in \mathbb{N}$ and applying Lemma 2, we obtain

$$
\frac{1}{p^{\nu}} \Delta\left(\frac{x}{p^{\nu}}\right)-g\left(p^{\nu}\right) \Delta(x)-\frac{1}{p^{\nu+1}} \sum_{\varrho=0}^{p-1} \Delta\left(\frac{p x+\varrho}{p^{\nu+1}}\right)=g\left(p^{\nu}\right) \Sigma_{p},
$$

for all $p \in \mathbb{P}$ and $\nu \in \mathbb{N}_{0}$, where

$$
\Sigma_{p}=\sum_{k=1}^{\infty} g^{-1}\left(p^{k}\right) \sum_{\delta=0}^{p^{k}-1} \Delta\left(p^{k} x+\delta\right) .
$$

In particular, for $\nu=0$ we see that

$$
\Sigma_{p}=-\frac{1}{p} \sum_{\varrho=0}^{p-1} \Delta\left(\frac{p x+\varrho}{p}\right),
$$

from which (13) follows with $p x$ instead of $x$.

The derivation of (14) and (15) from (12) and (13) requires two more lemmas concerning difference equations and will be given in the next section. 
Combining (14) and (15) gives

$$
\begin{aligned}
\sum_{s \in S}\left(\frac{x}{p}\right)^{s-1}\left(p^{-\nu s} P_{s}\left(\log x-\log p^{\nu+1}\right)-g\left(p^{\nu}\right)\right. & \left.P_{s}(\log x-\log p)\right) \\
& =p^{-\nu} \sum_{\zeta \in T_{p}} h_{\zeta, p, \nu}(x) \zeta^{x}
\end{aligned}
$$

Observe that $1 \in T_{p}$ and all $\zeta \in T_{p}, \zeta \neq 1$, satisfy $|\zeta|>1$. Therefore the right side of the above equation reduces to the term $p^{-\nu} h_{1, p, \nu}(x)=h_{p, \nu}(x)$, say, and with $p x$ instead of $x$ we arrive at

$$
\sum_{s \in S} x^{s-1}\left(p^{-\nu s} P_{s}\left(\log x-\log p^{\nu}\right)-g\left(p^{\nu}\right) P_{s}(\log x)\right)=h_{p, \nu}(p x),
$$

where $h_{p, \nu} \in R_{1}$. It follows from $\Delta(x) \not \equiv 0$ that $\Delta(x)=a x^{s-1}$ with some constants $a \neq 0, s \in S$. Moreover, $g\left(p^{\nu}\right)=p^{-\nu s}$ or $s=1$. Since $g \in \mathcal{M}$, the first equation is valid for all $p \in \mathbb{P}$ and $\nu \in \mathbb{N}$ if $s \neq 1$. If $s=1$ then we see from Lemma 4 that $F(x)=a B_{1}(x)+\Phi(x)$ with some function $\Phi \in R_{1}$. On inserting this into $\left(2_{n}\right)$ we obtain

$$
a \sum_{d=1}^{\infty} \lambda_{n}(d) B_{1}(d x)-\frac{a}{n} B_{1}(x)=\frac{1}{n} \sum_{\nu=0}^{n-1} \Phi\left(\frac{x+\nu}{n}\right)-\sum_{d=1}^{\infty} \lambda_{n}(d) \Phi(d x)
$$

where the right side represents a function $\Psi_{n} \in R_{1}$, say. Now $B_{1}(x)=x-1 / 2$ yields

$$
\sum_{d=1}^{\infty} \lambda_{n}(d) d=n^{-1}
$$

and

$$
\frac{a}{2}\left(\frac{1}{n}-f(n)\right)=\Psi_{n}(x),
$$

where, for abbreviation,

$$
f(n)=\sum_{d=1}^{\infty} \lambda_{n}(d) .
$$

By integrating over $\mathbb{T}$ we see that $\widehat{\Psi}_{n}(0)=\widehat{\Phi}(0)(1-f(n))$, leading to

$$
\frac{a}{2}\left(\frac{1}{n}-f(n)\right)=\widehat{\Phi}(0)(1-f(n)) .
$$

Suppose that $\widehat{\Phi}(0) \neq 0$. Then

$$
f(n)\left(\widehat{\Phi}(0)-\frac{a}{2}\right)=\widehat{\Phi}(0)-\frac{a}{2 n} \quad \text { and } \quad \widehat{\Phi}(0) \neq \frac{a}{2},
$$


so that

$$
f(n)=\frac{\widehat{\Phi}(0)-a /(2 n)}{\widehat{\Phi}(0)-a / 2} .
$$

By Lemma 3, $f$ is multiplicative, and we conclude from $f(m n)=f(m) f(n)$ for coprime $m, n \in \mathbb{N}$ that $\widehat{\Phi}(0)=0$, contrary to our assumption. Hence $\widehat{\Phi}(0)=0$ and $f(n)=n^{-1}$ by (17). Applying Lemma 3 again we obtain $g(n)=n^{-1}$, which is the desired conclusion.

EXAMPle 3. According to Example 2, the sequence $g=\left(\tau(n) n^{-s}\right)$ belongs to $\mathcal{M} \cap \mathcal{R}_{q}$ for every $q \in \mathbb{P}$. By Theorem 7 the corresponding system $\mathscr{S}$ has no aperiodic solutions $F \in C\left(\mathbb{R}_{+}\right)$. The same holds for the extended replicativity systems, generated for instance by the sequences $\left(\varphi(n) n^{-s}\right)$ and $\left(\sigma(n) n^{-s}\right)$.

6. Difference equations. In this section we close the gap in the preceding proof of Theorem 7 by deriving the equations (14) and (15) respectively from the functional equations (12) and (13). We begin with two lemmas concerning the continuous solutions of homogeneous difference equations.

For fixed $\omega \in \mathbb{R}_{+}$we are interested in the solutions $\eta \in C(\mathbb{R})$ of the linear homogeneous equation

$$
\eta(x+k \omega)+c_{k-1} \eta(x+(k-1) \omega)+\ldots+c_{0} \eta(x)=0 \quad(x \in \mathbb{R})
$$

where $k \in \mathbb{N}, c_{0}, \ldots, c_{k-1} \in \mathbb{C}, c_{0} \neq 0$. We may consider $C(\mathbb{R})$ as an $R_{\omega}$-module and interpret the indeterminate $z$ in the companion polynomial

$$
f(z)=z^{k}+c_{k-1} z^{k-1}+\ldots+c_{0} \in \mathbb{C}[z]
$$

of equation (18) as the endomorphism of $C(\mathbb{R})$ defined by $\eta \mapsto z \eta$ with $z \eta(x)=\eta(x+\omega)$ for all $x \in \mathbb{R}$. Further, we may write the composition of endomorphisms as product. Then (18) takes the form $f(z) \eta(x)=0$, and the problem of solving (18) consists in determining ker $f(z) \subseteq C(\mathbb{R})$. Observe that $\operatorname{ker} f(z)$ is a $k$-dimensional $R_{\omega}$-submodule of $C(\mathbb{R})$. Namely, if $\zeta_{1}, \ldots, \zeta_{r} \in \mathbb{C}^{\times}$are the distinct zeros of $f(z)$ of order $k_{1}, \ldots, k_{r} \in \mathbb{N}, k_{1}+$ $\ldots+k_{r}=k$, then

$$
\operatorname{ker} f(z)=\operatorname{ker}\left(z-\zeta_{1}\right)^{k_{1}} \oplus \ldots \oplus \operatorname{ker}\left(z-\zeta_{r}\right)^{k_{r}} .
$$

It remains to determine $\operatorname{ker}(z-\zeta)^{k}$ where $\zeta \in \mathbb{C}^{\times}, k \in \mathbb{N}$. Notice that the branches of the multi-valued function $\zeta^{x / \omega}=e^{(x / \omega) \log \zeta}$ only differ by a factor of the form $e(m x / \omega) \in R_{\omega}$ with $m \in \mathbb{Z}$. Now

$$
(z-\zeta)^{k} \zeta^{x / \omega} P(x)=\zeta^{k} \zeta^{x / \omega}(z-1)^{k} P(x)
$$

vanishes for all $x \in \mathbb{R}$ if and only if $(z-1)^{k} P(x) \equiv 0$, which is equivalent to $P(x) \in R_{\omega}[x], \operatorname{deg} P(x) \leq k-1$, or $P(x) \equiv 0$. We thus arrive at the following well known result (see, for instance, Nörlund [9], Chapter 10, no. 156). 
Lemma 6. Let $\zeta_{\varrho} \in \mathbb{C}^{\times}$be the distinct zeros of order $k_{\varrho} \in \mathbb{N}(\varrho=1, \ldots, r)$ of the companion polynomial (19), associated with equation (18). Then $\eta \in$ $C(\mathbb{R})$ solves $(18)$ if and only if

$$
\eta(x)=\sum_{1 \leq \varrho \leq r} P_{\varrho}(x) \zeta_{\varrho}^{x / \omega}
$$

with polynomials $P_{\varrho}(x) \in R_{\omega}[x], \operatorname{deg} P_{\varrho}(x) \leq k_{\varrho}-1$ or $P_{\varrho}(x) \equiv 0(\varrho=$ $1, \ldots, r)$.

In the sequel, we indicate the dependency on $\omega \in \mathbb{R}_{+}$of the shift operator $z$ defined above by writing $z_{\omega}$. The following lemma extends a theorem of Popoviciu [10] (see Kuczma [4], Theorem 13.5).

Lemma 7. Let $\omega_{1}, \omega_{2} \in \mathbb{R}_{+}$such that $\omega_{1} / \omega_{2} \notin \mathbb{Q}$. Let $f_{1}(z), f_{2}(z) \in \mathbb{C}[z]$ satisfy $f_{1}(0) f_{2}(0) \neq 0$, and let $\mathrm{A}$ be the (finite) set of (uniquely determined) solutions $\alpha \in \mathbb{C}$ of the system

$$
f_{1}\left(e^{\alpha \omega_{1}}\right)=f_{2}\left(e^{\alpha \omega_{2}}\right)=0 .
$$

Then every $\eta \in \operatorname{ker} f_{1}\left(z_{\omega_{1}}\right) \cap \operatorname{ker} f_{2}\left(z_{\omega_{2}}\right)$ has the form

$$
\eta(x)=\sum_{\alpha \in \mathrm{A}} P_{\alpha}(x) e^{\alpha x}
$$

with certain polynomials $P_{\alpha}(x) \in \mathbb{C}[x]$.

P r o o f. Because of the direct sum representation of the kernels of $f_{1}\left(z_{\omega_{1}}\right)$ and $f_{2}\left(z_{\omega_{2}}\right)$ it suffices to prove the following special case: Let $\zeta_{1}, \zeta_{2} \in \mathbb{C}^{\times}$ and $k \in \mathbb{N}$. Then the existence of a non-trivial solution $\eta \in C(\mathbb{R})$ of the difference equations

$$
\left(z_{\omega_{1}}-\zeta_{1}\right)^{k} \eta=0, \quad\left(z_{\omega_{2}}-\zeta_{2}\right)^{k} \eta=0
$$

implies that $\zeta_{1}=e^{\alpha \omega_{1}}, \zeta_{2}=e^{\alpha \omega_{2}}$ with a uniquely determined number $\alpha \in \mathbb{C}$, and $\eta(x)=P(x) e^{\alpha x}$ with some polynomial $P(x) \in \mathbb{C}[x], \operatorname{deg} P(x) \leq k-1$.

Fix some values $\alpha_{1}$ of $\left(\log \zeta_{1}\right) / \omega_{1}$ and $\alpha_{2}$ of $\left(\log \zeta_{2}\right) / \omega_{2}$. Then, by the irrationality of $\omega_{1} / \omega_{2}$, the equation

$$
\alpha_{1}+\frac{2 m_{1} \pi i}{\omega_{1}}=\alpha_{2}+\frac{2 m_{2} \pi i}{\omega_{2}}
$$

has at most one solution $\left(m_{1}, m_{2}\right) \in \mathbb{Z}^{2}$.

Lemma 6 yields the representations

$$
\eta(x)=P_{1}(x) e^{\alpha_{1} x}=P_{2}(x) e^{\alpha_{2} x} \quad(x \in \mathbb{R})
$$

with polynomials $P_{1}(x) \in R_{\omega_{1}}[x], P_{2}(x) \in R_{\omega_{2}}[x]$ of degree $\leq k-1$. By comparing summands we obtain

$$
h_{1 \kappa}(x) x^{\kappa} e^{\alpha_{1} x}=h_{2 \kappa}(x) x^{\kappa} e^{\alpha_{2} x} \quad(x \in \mathbb{R}, 0 \leq \kappa<k)
$$


where $h_{1 \kappa} \in R_{\omega_{1}}, h_{2 \kappa} \in R_{\omega_{2}}$. In particular, with $h=h_{1 \kappa}$ and $\delta=\alpha_{2}-\alpha_{1}$, it follows that $h\left(x+\omega_{1}\right)=h(x)$ and $h\left(x+\omega_{2}\right)=e^{\delta \omega_{2}} h(x)$ for all $x \in \mathbb{R}$ or, equivalently,

$$
h\left(x+m \omega_{1}+n \omega_{2}\right)=e^{n \delta \omega_{2}} h(x) \quad(m, n \in \mathbb{Z}, x \in \mathbb{R}) .
$$

By Kronecker's theorem (see for instance Hardy and Wright [2], Theorem 444) the set $\left\{m \omega_{1}+n \omega_{2}: m, n \in \mathbb{Z}\right\}$ is dense in $\mathbb{R}$. Consequently, for every $y \in \mathbb{R}$ there exists a sequence of pairs $\left(m_{\nu}, n_{\nu}\right) \in \mathbb{Z}^{2}$ depending only on $y$ such that the corresponding sequence $\left(m_{\nu} \omega_{1}+n_{\nu} \omega_{2}\right)$ tends to $y$ as $\nu$ tends to infinity. Since $h$ is continuous, (22) implies

$$
h(x+y)=h(x) H(y) \quad(x, y \in \mathbb{R})
$$

with some function $H: \mathbb{R} \rightarrow \mathbb{C}$. We may assume that $h(x) \not \equiv 0$. Then $h$ is zerofree on $\mathbb{R}$ and $h(y)=h(0) H(y)$. Therefore, $H$ is continuous and zerofree too, and additionally satisfies the functional equation

$$
H(x+y)=H(x) H(y) \quad(x, y \in \mathbb{R}) .
$$

These functions are given by $H(x)=e^{\beta x}$, which leads to $h(x)=c e^{\beta x}$, with some constants $\beta \in \mathbb{C}, c \in \mathbb{C}^{\times}$. By inserting we see from (22) that $e^{\beta \omega_{1}}=1$ and $e^{\beta \omega_{2}}=e^{\delta \omega_{2}}$ or, equivalently, $\beta \omega_{1}=2 \varrho_{1} \pi i$ and $\beta \omega_{2}=\delta \omega_{2}+2 \varrho_{2} \pi i$ with certain numbers $\varrho_{1}, \varrho_{2} \in \mathbb{Z}$. It follows that

$$
\delta=\frac{2 k \pi i}{\omega_{1}}-\frac{2 l \pi i}{\omega_{2}}
$$

which shows that (20) has at least one solution. We conclude that $\alpha$ is well defined.

Actually, the equations (20), (21) and (23) yield that the function defined by

$$
x \mapsto h_{1 \kappa}(x) e\left(-\frac{\varrho_{1}}{\omega_{1}} x\right)=h_{2 \kappa}(x) e\left(-\frac{\varrho_{2}}{\omega_{2}} x\right) \quad(x \in \mathbb{R})
$$

belongs to $R_{\omega_{1}} \cap R_{\omega_{2}}$ and therefore must be a constant. This means that $\eta(x)=P(x) e^{\alpha x}$ with a polynomial $P(x) \in \mathbb{C}[x]$ of degree $\leq k-1$, which is the desired conclusion.

In order to derive (14) from (12) we multiply (12) with $x$, replace $x$ by $e^{x}$, put $\eta(x)=e^{x} \Delta\left(e^{x}\right)$ and $\omega=\log q$. This yields the difference equation

$$
\eta(x-k \omega)+c_{k-1} \eta(x-(k-1) \omega)+\ldots+c_{0} \eta(x)=0 \quad(x \in \mathbb{R}) .
$$

With the companion polynomial $f_{q}(z)$ of $g \in \mathcal{R}_{q}$ according to (6), the companion polynomial of equation (24) equals $z^{k} f_{q}(1 / z)$. Therefore the zeros $\zeta_{\varrho} \in Z_{q}(\varrho=1, \ldots, r)$ of order $k_{\varrho} \in \mathbb{N}$ of $f_{q}(z)$ correspond to the zeros $\zeta_{\varrho}^{-1} \in \mathbb{C}^{\times}$of the same order of $z^{k} f_{q}(1 / z)$. By applying Lemma 6 and 
returning to $\Delta$ we obtain

$$
\Delta(x)=\sum_{1 \leq \varrho \leq r} P_{\varrho}(\log x) x^{s_{\varrho}-1} \quad\left(x \in \mathbb{R}_{+}\right)
$$

with polynomials $P_{\varrho}(x) \in R_{\omega}[x], \operatorname{deg} P_{\varrho}(x) \leq k_{\varrho}-1$, or $P_{\varrho}(x) \equiv 0$, where $s_{\varrho}$ is some fixed value of $-\left(\log \zeta_{\varrho}\right) / \omega$ for $\varrho=1, \ldots, r$.

Now we associate with $g \in \mathcal{R}_{q}$ the set $S_{q}=\left\{s \in \mathbb{C}: f_{q}\left(q^{-s}\right)=0\right\}$. Observe that $S_{q}=\emptyset$ if and only if $k=0$, and $\operatorname{card} S_{q}=\infty$ for $k \in \mathbb{N}$, since every zero $\zeta \in Z_{q}$ of the companion polynomial $f_{q}(z)$ contributes the distinct elements

$$
-\frac{\log \zeta+2 m \pi i}{\log q} \quad(m \in \mathbb{Z})
$$

to $S_{q}$, where $\log \zeta$ is some fixed logarithm of $\zeta \neq 0$. Assume now that $g \in$ $\mathcal{R}_{q_{1}} \cap \mathcal{R}_{q_{2}}, g(1)=1$, with $\omega_{1}=\log q_{1}$ and $\omega_{2}=\log q_{2}$ linearly independent over $\mathbb{Q}$. Due to the irrationality of $\omega_{1} / \omega_{2}$, the intersection $S=S_{q_{1}} \cap S_{q_{2}}$ is always a finite set. Notice that for every $s \in S$ the numbers $\zeta_{1}=e^{-\omega_{1} s}$, $\zeta_{2}=e^{-\omega_{2} s}$ are zeros of $f_{q_{1}}(z)$ and $f_{q_{2}}(z)$, respectively. Hence, by applying Lemma 7 to the (transformed) functional equations (12) for $q=q_{1}, q_{2}$ we obtain (14).

In order to derive (15) from (13) denote the left hand side of (13) by $H(x)$. Then (13) takes the form of the difference equation

$$
H(x)=\frac{1}{p} \sum_{\varrho=0}^{p-1} H(x+\varrho) \quad\left(x \in \mathbb{R}_{+}\right)
$$

with the companion polynomial $t_{p}(z)=\left(z^{p}-1\right) /(z-1)-p$ having only simple zeros $\zeta \in T_{p}$. According to Lemma 6 , the solutions $H \in C\left(\mathbb{R}_{+}\right)$are given by $H(x)=\sum_{\zeta \in T_{p}} h_{\zeta}(x) \zeta^{x}$ with functions $h_{\zeta} \in R_{1}$, which gives (15) and completes the proof of Theorem 7 .

A closer inspection of the proof of Theorem 7 shows that the existence of aperiodic solutions $F \in C\left(\mathbb{R}_{+}\right)$of certain extended replicativity systems $\mathscr{S}$ can be excluded by considering suitable finite subsystems only. This requires looking again at the companion polynomial

$$
f_{q}(z)=c_{k} z^{k}+c_{k-1} z^{k-1}+\ldots+c_{0} \in \mathbb{C}[z]
$$

of $g \in \mathcal{R}_{q}$, where $c_{k}=1$ and $c_{0} \neq 0$. We associate with $g \in \mathcal{R}_{q}$ the (non-empty) set

$$
N_{q}=\left\{q^{l+\kappa}: 0 \leq \kappa \leq k, c_{\kappa} \neq 0\right\}
$$

of powers of $q$, where $k, l \in \mathbb{N}_{0}$ are chosen minimal such that equation $\left(5_{q}\right)$ is valid.

THEOREM 8. For different primes $q_{1}, q_{2}$ assume that the sequence $g \in \mathcal{F}$, normalized by $g(1)=1$, satisfies $g \in \mathcal{R}_{q}$ and $\operatorname{supp} \lambda_{n} \subseteq\langle q\rangle$ for all $n \in\langle q\rangle$, 
$q \in\left\{q_{1}, q_{2}\right\}$. If the system $\mathscr{S}$ generated by $g$ has aperiodic solutions $F \in$ $C\left(\mathbb{R}_{+}\right)$then there exists some $s \in \mathbb{C}$ such that $g(n)=n^{-s}$ for all $n \in\left\langle q_{1} q_{2}\right\rangle$.

Hence, if the finite subsystem of $\mathscr{S}$ consisting of the equations $\left(2_{n}\right)$ for all $n \in N_{q_{1}} \cup N_{q_{2}}$ does not coincide with that of the classical system generated

by $g(n)=n^{-s}$ then $\mathscr{S}$ has no aperiodic solutions $F \in C\left(\mathbb{R}_{+}\right)$. For the proof of Theorem 8 we refer to that of Theorem 7 .

ExAmple 4. According to Example 2, the non-existence of aperiodic solutions $F \in C\left(\mathbb{R}_{+}\right)$of the system $\mathscr{S}$ generated by $\left(\tau(n) n^{-s}\right)$ follows already from its equations $\left(2_{n}\right)$ for $n=q, q^{2}$ and two different primes $q$.

\section{References}

[1] E. Artin, The Gamma Function, Holt, Rinehart and Winston, New York, 1964.

[2] G. H. Hardy and E. M. Wright, An Introduction to the Theory of Numbers, 3rd ed., Oxford Univ. Press, London, 1960.

[3] D. Ku bert, The universal ordinary distribution, Bull. Soc. Math. France 107 (1979), 179-202.

[4] M. Kuczma, Functional Equations in a Single Variable, PWN-Polish Scientific Publishers, Warszawa, 1968.

[5] R. Lidl and H. Niederreiter, Introduction to Finite Fields and their Applications, Cambridge Univ. Press, London, 1986.

[6] L. G. Lucht, Arithmetical sequences and systems of functional equations, Aequationes Math. 53 (1997), 73-90.

[7] C. Methfessel, Multiplicative and additive recurrent sequences, Arch. Math. (Basel) 63 (1994), 321-328.

[8] J. Milnor, On polylogarithms, Hurwitz zeta functions, and the Kubert identities, Enseign. Math. 29 (1983), 281-322.

[9] N. E. Nörlund, Vorlesungen über Differenzenrechnung, Springer, Berlin, 1924.

[10] T. Popoviciu, Remarques sur la définition fonctionnelle d'un polynôme d'une variable réelle, Mathematica (Cluj) 12 (1936), 5-12.

[11] W. Rudin, Real and Complex Analysis, McGraw-Hill, London, 1970.

[12] M. F. Yoder, Continuous replicative functions, Aequationes Math. 13 (1975), 251261.

Institut für Mathematik

Technische Universität Clausthal

Erzstrasse 1

38678 Clausthal-Zellerfeld, Germany

E-mail: lucht@math.tu-clausthal.de 